\title{
Avaliação de estudantes de enfermagem sobre o exame clínico objetivamente estruturado
}

\section{Evaluation of nursing students about the objective structured clinical examination}

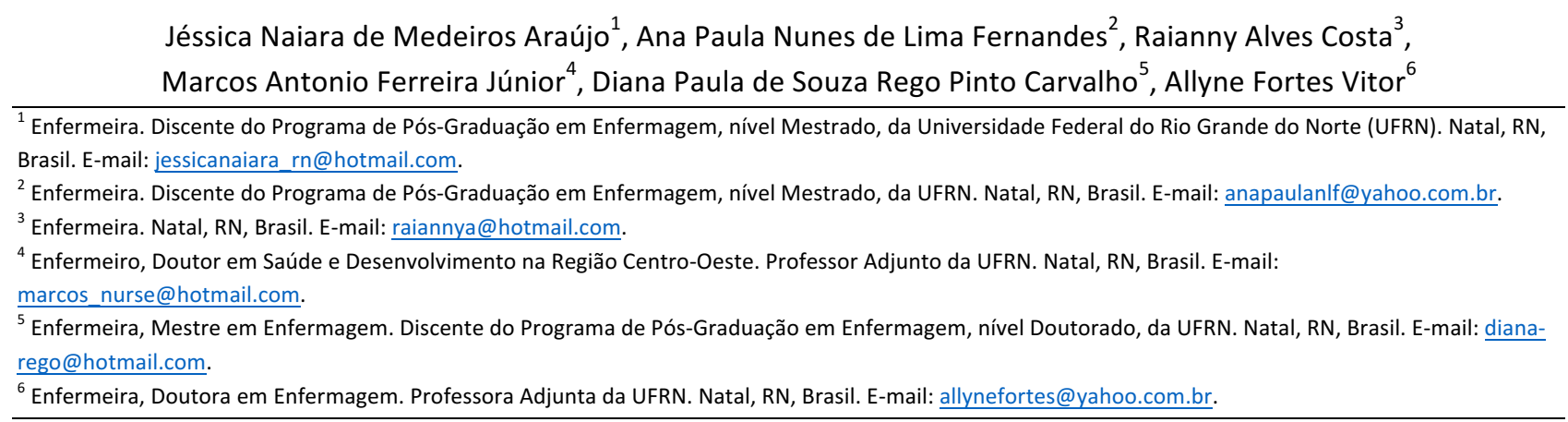

\section{RESUMO}

O Exame Clínico Objetivamente Estruturado (Objective Structured Clinical Examination/OSCE) é apontado como elemento fundamental na melhoria do ensino-aprendizagem. Pode ser compreendido como ferramenta de mensuração de competências clínicas com adoção de procedimentos padronizados. Estudo qualitativo objetivou descrever a avaliação dos estudantes sobre o uso do OSCE como estratégia de promoção do processo de ensino-aprendizagem em Enfermagem Clínica. Foi formado um grupo focal de 27 estudantes para a coleta de dados com informações extraídas a partir de três questões norteadoras. Os dados foram analisados mediante a técnica de Análise de Conteúdo. Com a primeira questão norteadora surgiram duas categorias: utilização do POP; e estratégia de aprendizagem. Mediante a segunda questão elencou-se três categorias: principais sentimentos; experiência válida; e método avaliativo. Com a terceira identificou-se duas categorias: aprender com os erros; e semelhança com a prática. O OSCE constitui uma estratégia avaliativa que contribuiu positivamente no processo de ensino-aprendizagem em Enfermagem Clínica.

Descritores: Simulação; Aprendizagem; Materiais de Ensino; Educação em Enfermagem.

\section{ABSTRACT}

The Objective Structured Clinical Examination (OSCE) is considered a fundamental element to improve teaching and learning. It can be understood as a measurement tool of clinical competencies with adoption of standard procedures. A qualitative study aimed to describe the evaluation of students about the OSCE use as strategy to promote the teaching-learning process in Clinical Nursing. A focus group with 27 students was created for data collection with information extracted from three guiding questions. Data was analyzed using Content Analysis technique. Two categories emerged from the first guiding question: the use of SOP; and learning strategy. Three categories emerged from the second question: main feelings; valid experience; and assessment method. From the third question, two categories were identified: learning from errors; and similarity with practice. The OSCE constitutes an assessment strategy that positively contributed with the teaching-learning process in Clinical Nursing.

Descriptors: Simulation; Learning; Teaching Materials; Education, Nursing. 


\section{INTRODUÇÃO}

O processo de ensino-aprendizagem em enfermagem deve estimular as práticas de atenção à saúde e estabelecer uma educação crítica-reflexiva, com aquisição de conhecimentos, habilidades e atitudes que tornem os estudantes capazes de atuar nas práticas hospitalares. Assim, torna-se essencial que o currículo dos cursos de enfermagem considere atividades práticas e teóricas ${ }^{(1)}$.

Há uma forte tendência na inserção dos estudantes em ações práticas de forma precoce, pois em um cenário de aprendizado, estes sentem dificuldades em recordar conhecimentos adquiridos anteriormente em aulas teóricas e mostram-se inseguros para aplicá-los na prática. Esta problemática advém da dissociação entre o aprendizado clínico e o ambiente/ situação em que ele será aplicado ${ }^{(2)}$.

O Exame Clínico Objetivamente Estruturado (OSCE, do inglês, Objective Structured Clinical Examination) é apontado como elemento fundamental na melhoria do ensino-aprendizagem, trata-se de uma ferramenta para mensuração de competências clínicas com a adoção de procedimentos padronizados. Além disso, resgata o conhecimento prévio adquirido pelo estudante, ampliando as oportunidades de aprendizagem em um cenário clínico ${ }^{(3)}$.

O OSCE foi aplicado pioneiramente na medicina em 1975 por Harden, principalmente nos Estados Unidos ${ }^{(4)}$. Nos cursos de enfermagem, de maneira mais recente, é realizado em outros países, como o Chile, Egito, Irlanda, Reino Unido e Estados Unidos ${ }^{(1,5-9)}$. No Brasil, esta estratégia é utilizada na medicina pela primeira vez, na faculdade de Marília, já na farmácia, por uma universidade do nordeste em $1990^{(3,10-11)}$. Na enfermagem começou a ser utilizada recentemente, segundo um relato de experiência no qual se aplicou o OSCE em uma universidade do Rio Grande do Norte ${ }^{(12)}$.

Aplicar a simulação de casos clínicos reais na formação acadêmica significa abranger um enfoque problematizador que auxilia, sobretudo, na construção do aprendizado $^{(3)}$. Como forma de aquisição de conhecimento, a utilização do OSCE torna-se importante, uma vez que os professores posicionam o estudante frente a situações reais de tomadas de decisões e condutas de enfermagem.

Consiste em uma maneira de avaliar o raciocínio clínico. Estrutura-se em estações, as quais apresentam um tempo pré-determinado para seu desenvolvimento, formam um circuito em que os estudantes se dispõem em forma rotativa e simultânea a uma multiplicidade de assuntos, cujos temas dependem dos objetivos de aprendizagem $^{(1,13)}$.

Como instrumento metodológico para execução do OSCE, utiliza-se o Procedimento/protocolo Operacional Padrão (POP), o qual expõe cada passo sequencial que o estudante deve realizar. A intenção dos POPs é orientar o desempenho de procedimentos e elucidar dúvidas, devem ser atualizados e seguidos pelos estudantes de forma padronizada ${ }^{(14)}$.

As simulações em laboratórios com a utilização do OSCE promovem ao estudante uma aproximação com situações reais que serão vivenciadas nos ambientes de atenção à saúde. Deste modo, torna-se fundamental compreender a utilização desta estratégia como ferramenta de ensino-aprendizagem. Tal compreensão pode servir como guia para o uso desta estratégia no processo de formação dos estudantes. Destaca-se que existem escassos estudos no Brasil que avaliem o uso do OSCE na formação do profissional enfermeiro.

Diante da utilização desta estratégia, surge o seguinte questionamento de pesquisa: Qual a avaliação dos estudantes sobre o uso do OSCE como estratégia de melhoria para o processo de ensino-aprendizagem?

Para tanto, este estudo objetiva descrever a avaliação dos estudantes sobre o uso do OSCE como estratégia de promoção do processo de ensino-aprendizagem em Enfermagem Clínica.

\section{MÉTODO}


Trata-se de um estudo descritivo, com abordagem qualitativa sobre a avaliação do OSCE no ensinoaprendizagem em Enfermagem Clínica do curso de graduação em Enfermagem de uma universidade pública localizada no nordeste brasileiro. Este estudo foi realizado no Departamento de Enfermagem da referida universidade.

A população foi composta pelos 46 estudantes que cursaram o sétimo período do curso de graduação em Enfermagem, que já haviam cursado a disciplina de Enfermagem Clínica, que passou a adotar a partir de 2013 - OSCE como estratégia de ensino e avaliação da aprendizagem. Na ocasião foi formado um grupo focal para a coleta de dados. Foram incluídos os estudantes que haviam cursado a referida disciplina no período de 2013.2 e que aceitaram participar da pesquisa.

Foram excluídos os que estiveram ausentes no dia da realização da coleta de dados e que não participaram da avaliação com uso do OSCE. A amostra foi constituída de forma intencional, uma vez que os sujeitos foram escolhidos de acordo com os objetivos da pesquisa, constituída pelos estudantes que aceitaram participar do grupo focal e assinaram o Termo de Consentimento Livre e Esclarecido (TCLE) e o Termo de autorização para uso de voz, com obtenção de um total de 27 participantes denominados sujeitos ${ }^{(15)}$.

A coleta de dados ocorreu no mês de agosto de 2014 com uso de questões sobre o tema proposto que nortearam as atividades do grupo focal. As falas foram gravadas e em seguida realizada a análise. As questões norteadoras foram elaboradas de acordo com os objetivos do estudo, a saber: 1) OSCE: Qual a sua opinião sobre a aplicação desta estratégia de ensino em Enfermagem Clínica? 2) Como foi sua experiência? 3) Você acha que obteve ou irá obter melhor desempenho nas atividades práticas hospitalares com o uso desta estratégia? De que forma?

Os dados foram analisados por meio da técnica de Análise de Conteúdo, que incluiu as seguintes etapas: a pré-análise, a inferência e a interpretação. Destaca-se que a categorização ocorreu à posteriori ${ }^{(16)}$. Foram obedecidas as determinações da Resolução no 466/2012 do Conselho Nacional de Saúde ${ }^{(17)}$. Salienta-se que este estudo obteve aprovação do Comitê de Ética em Pesquisa sob parecer no 617.576 e CAAE $\mathrm{n}$ 은 28667714.3.0000.5537.

\section{RESULTADOS}

Inicialmente os participantes foram indagados sobre o que seria o OSCE, apesar de não ter sido uma das questões norteadoras, foi uma forma encontrada pelas pesquisadoras de introduzi-los mais sutilmente na discussão e deixá-los mais seguros durante os próximos questionamentos. Obteve-se a seguinte resposta pela maioria dos participantes: O OSCE seria uma forma prática para avaliar nossos conhecimentos, para testar 0 que a gente aprendeu na teoria para pôr em prática.

O primeiro questionamento foi sobre a opinião dos estudantes em relação à utilização do OSCE como estratégia de ensino em Enfermagem Clínica, assunto tratado na disciplina de Enfermagem Clínica, na qual o OSCE é aplicado. Com este questionamento surgiram duas categorias que foram demonstradas no Quadro 1. 
Quadro 1: Categorias das respostas sobre o questionamento: OSCE: qual a sua opinião sobre a aplicação desta estratégia de ensino em Enfermagem Clínica? Natal, RN, Brasil, 2014.

\begin{tabular}{|c|c|}
\hline CATEGORIAS & RESPOSTAS \\
\hline \multirow[t]{3}{*}{ Utilização do POP } & $\begin{array}{c}\text { É fundamental, porque a gente sentiu a diferença, nesse período a gente teve uma espécie de } \\
\text { prova prática, não sei se foi o OSCE propriamente dito, e a gente sentiu a diferença que faz de } \\
\text { não ter um POP que é o procedimento operacional padrão para a gente seguir aquelas regras } \\
\text { de acordo com o que é preconizado. É fundamental, foi difícil sem esse POP para o } \\
\text { desenvolvimento do OSCE. (Sujeito 3) }\end{array}$ \\
\hline & $\begin{array}{l}\text { O atendimento fica bem mais organizado, porque você estrutura na sua cabeça o que você vai } \\
\text { fazer e como vai fazer, o que você pode perguntar, então fica bem mais fácil para a gente } \\
\text { essa organização de acordo com o POP. (Sujeito 5) }\end{array}$ \\
\hline & Neste caso, fica também alguns pontos negativos, porque é muito decoreba. (Sujeito 6) \\
\hline \multirow{5}{*}{ Estratégia de aprendizagem } & $\begin{array}{l}\text { Com relação ao OSCE, é válido para a gente aprender e realmente fixar aquilo que foi dado } \\
\text { em sala de aula. (Sujeito 7) }\end{array}$ \\
\hline & $\begin{array}{c}\text { O OSCE é bem válido, mas não deveria ser uma forma de avaliação, ele pode estar inserido, } \\
\text { mas sem precisar valer ponto, porque mexe totalmente com o emocional e principalmente } \\
\text { para aquelas que não saber lidar sob pressão. (Sujeito 8) }\end{array}$ \\
\hline & $\begin{array}{l}\text { Eu acho que isso é uma coisa muito relativa, porque se não for nessa pressão, a gente não dá } \\
\text { importância, tem que ter muita disciplina. (Sujeito 17) }\end{array}$ \\
\hline & $\begin{array}{l}\text { O OSCE acaba sendo uma experiência, porque na prática a gente vai lidar com essa pressão, } \\
\text { então no OSCE a gente também está sob pressão. (Sujeito 23) }\end{array}$ \\
\hline & O OSCE é uma ferramenta importantíssima para o aprendizado da gente. (Sujeito 16) \\
\hline
\end{tabular}

O segundo questionamento abordou a experiência dos participantes com a utilização desta estratégia de ensino. No momento, os estudantes estavam bem expressivos e demonstraram principalmente os sentimentos observados durante a avaliação da disciplina com o uso do OSCE. Dessa questão surgiram três categorias demonstradas no Quadro 2.

Quadro 2: Categorias das respostas sobre o questionamento: Como foi sua experiência? Natal, RN, Brasil, 2014.

\begin{tabular}{|c|c|}
\hline CATEGORIAS & RESPOSTAS \\
\hline \multirow[b]{2}{*}{ Principais sentimentos } & Ansiedade, nervosismo, medo. (Sujeito 9) \\
\hline & $\begin{array}{c}\text { Frustração, choro, desilusão. Porém é algo que nos norteia na prática. Temos que ver os dois } \\
\text { lados. (Sujeito 10) }\end{array}$ \\
\hline \multirow[b]{2}{*}{ Experiência Válida } & $\begin{array}{c}\text { Foi uma experiência bastante válida principalmente para a gente não chegar na prática sem } \\
\text { saber nada, sem ter feito nenhum procedimento. Então acho que foi válida em todas as } \\
\text { vertentes. (Sujeito 3) }\end{array}$ \\
\hline & $\begin{array}{c}\text { E eu acho que é importante também, porque embora você fique muito nervosa, mas é um } \\
\text { primeiro contato que você tem, ali você pode estar errando e isso facilita para quando você } \\
\text { for prestar essa assistência ao paciente, porque você já vai estar suprido das necessidades } \\
\text { que você teve no OSCE. (Sujeito 14) }\end{array}$ \\
\hline \multirow{4}{*}{ Método avaliativo } & $\begin{array}{l}\text { Talvez seria interessante nas avaliações, não ser o professor que esteja avaliando, mas sim } \\
\text { pessoas desconhecidas que possam te avaliar tão bem quanto um professor. (Sujeito 13) }\end{array}$ \\
\hline & $\begin{array}{c}\text { Você estuda, sabe a sequência e tudo mais, porém como ela falou, por essa questão de valer } \\
\text { nota, porque o professor está ali olhando para você, eu acho que por eles estarem presentes } \\
\text { naquele momento, eu acho que é o que mexe mais. (Sujeito 11) }\end{array}$ \\
\hline & $\begin{array}{l}\text { Você fica tão bitolado no tempo, estuda em casa, vai cronometrar e quando você chega aqui } \\
\text { (local da avaliação) você já esqueceu tudo. Aí quando você entra na sala de aula que você dá } \\
\text { de cara com os professores, aí é que você não lembra nada mesmo. (Sujeito 12) }\end{array}$ \\
\hline & Acho que por ser uma forma avaliativa, acaba atrapalhando. (Sujeito 16) \\
\hline
\end{tabular}

A terceira e última questão foi sobre a aquisição de melhor desempenho nas atividades práticas hospitalares com o uso desta estratégia. Todos os participantes do grupo focal responderam que "sim". A partir desta questão norteadora, surgiram duas categorias de acordo com o Quadro 3. 
Quadro 3. Categorias das respostas sobre o questionamento: Você acha que obteve ou irá obter melhor desempenho nas atividades práticas hospitalares com o uso desta estratégia? De que forma? Natal, RN, Brasil, 2014.

\begin{tabular}{|c|c|}
\hline CATEGORIAS & RESPOSTAS \\
\hline \multirow{2}{*}{ Aprender com os erros } & $\begin{array}{c}\text { Com certeza, porque você está estudando, aprende com os erros e quando chegar na prática, } \\
\text { eu vou me lembrar de tudo que eu passei, de tudo que eu estudei. (Sujeito 21) }\end{array}$ \\
\hline & $\begin{array}{l}\text { Os erros que a gente obtém lá no momento do OSCE, a gente conserta para que em um } \\
\text { momento oportuno de que não pode errar, a gente consegue lembrar. (Sujeito 4) }\end{array}$ \\
\hline \multirow{5}{*}{ Semelhança com a prática } & $\begin{array}{c}\text { No OSCE, a gente lidava com situações em que o professor ele se colocava como paciente, } \\
\text { então ele poderia mudar alguns aspectos, e isso na prática a gente vê muito, a pessoa é muito } \\
\text { dinâmica, a gente não vai lidar com uma coisa programada. (Sujeito 17) }\end{array}$ \\
\hline & $\begin{array}{c}\text { No estágio, eu peguei uma situação parecida com a abordada no OSCE, aí eu fui levar em } \\
\text { consideração ao que eu tinha visto no OSCE, e me ajudou bastante, porque que você monta } \\
\text { na sua cabeça uma sequência. (Sujeito 7) }\end{array}$ \\
\hline & $\begin{array}{c}\text { Por isso que acho que também não se deve falar mal da decoreba, porque o procedimento, } \\
\text { querendo ou não, segue um padrão, segue uma sequência mesmo. (Sujeito 24) }\end{array}$ \\
\hline & $\begin{array}{c}\text { Uma das coisas mais válidas do OSCE, é quando a gente vai para a prática, porque o medo é } \\
\text { de lidar com uma situação, não saber organizar, não saber o que será imposto para a gente, e } \\
\text { no OSCE, por mais que seja uma situação simulada, a gente está vendo como vai ser na } \\
\text { prática, a gente sabe como se impor diante de uma situação. Esse pra mim é um dos pontos } \\
\text { mais positivos em relação ao OSCE. (Sujeito 19) }\end{array}$ \\
\hline & $\begin{array}{c}\text { E também a gente tem que ver o futuro, porque no futuro a gente vai trabalhar em algum } \\
\text { lugar, e esses lugares têm seus protocolos e a gente deve segui-los, então se a gente já tem } \\
\text { estruturado na cabeça essa experiência acadêmica, vai ser bem mais fácil para quando a } \\
\text { gente chegar lá e conseguir lembrar também ou organizar de acordo com a instituição que a } \\
\text { gente vai trabalhar. (Sujeito 21) }\end{array}$ \\
\hline
\end{tabular}

\section{DISCUSSÃO}

Concernente à primeira fala dos estudantes sobre o que seria o OSCE, um relato de experiência confirmou que este método é uma ferramenta de medida de competências clínicas com a utilização de procedimentos e pacientes padronizados. É considerado pelos estudantes de suma importância para entrada nos campos de prática, uma vez que os auxiliam a desenvolver habilidades indispensáveis ao atendimento clínico ${ }^{(3)}$.

De acordo com o Quadro 1, com o primeiro questionamento surgiram duas categorias, a primeira foi sobre a utilização do POP, a qual obteve pontos positivos e negativos. Sobre isto, outro estudo ${ }^{(18)}$ descreve que o POP tem o objetivo de esclarecer dúvidas, padronizar a execução de ações e orientar de acordo com as normas de cada instituição, o qual deve ser atualizado e seguido por todos de forma uniformizada. Além disso, descreve cada passo para promover um desempenho adequado ${ }^{(18-}$ 19).

De acordo com autores de um estudo sobre o ponto de vista da importância dos POPs para pesquisa clínica, este instrumento deverá ser desenvolvido por uma equipe específica, determinada para este fim, esta poderá avaliar e validar os procedimentos ou contratar um grupo com esta finalidade ${ }^{(19)}$.

Os POPs são recursos importantes na prática de saúde, pois ajudam a sintetizar a informação de acordo com uma estrutura concisa e desenvolve uma melhoria no ambiente prático ${ }^{(18)}$. Por ser uma ferramenta que segue um padrão sequencial, podem surgir com este instrumento metodológico alguns pontos negativos, como o fato de "decorar", relatado por alguns estudantes, por ser um momento em que será avaliado, desse modo, gera-se uma apreensão, porém, isto é muito relativo de acordo com a opinião de cada estudante, pôde ser observado por uma minoria.

Grande parte dos estudantes elencou o POP como uma forma positiva a ser usado no OSCE, relativo a esta informação, autores de um estudo bibliográfico com análise integrativa relataram o POP como uma ferramenta essencial na área da saúde ${ }^{(14)}$.

Conforme o Quadro 2, a segunda categoria do primeiro questionamento abordou a estratégia de aprendizagem como de extrema importância para a 
melhoria do aprendizado. Quanto a esta categoria, autores de uma reflexão teórica referem que as atividades práticas simuladas em laboratório é um espaço essencial de aprendizagem, pois além de facilitá-la, influencia os estudantes a continuarem a aprender, além disso, desenvolve nestes a criatividade e sensibilidade, ademais outros aspectos indispensáveis no ato de educar e aprender ${ }^{(20)}$.

Além do mais, é considerado um fator de melhoria no desempenho profissional, uma vez que a simulação não oferece risco ou desconforto aos pacientes. Das várias estratégias de ensino, as que garantem oportunidade de treino prático e aquisição de habilidades são apontadas como essenciais ${ }^{(20)}$. O OSCE reproduz as ações reais que um profissional deve executar em determinada situação clínica $^{(21)}$.

Consoante com tal percepção, outra pesquisa ${ }^{(3)}$ descreve que o OSCE é tanto uma ferramenta de avaliação quanto uma atividade educativa, pois auxilia o estudante a desenvolver habilidades necessárias durante o atendimento clínico e ajuda na identificação de lacunas no processo de ensino-aprendizagem.

Com a segunda questão norteadora foram elencadas três categorias. A primeira englobou os principais sentimentos expressos pelos estudantes no momento da avaliação. Os estudantes relataram que no momento sentiram "Ansiedade, nervosismo, medo, frustração, choro e desilusão", porém concordaram que era algo que norteava a prática. A favor disto, um estudo transversal sobre a avaliação clínica objetiva e estruturada como um método para avaliar estudantes de graduação em fisioterapia respiratória, comenta que o OSCE é o tipo mais estressante de exame para os estudantes, e o estresse pode afetar adversamente o desempenho ${ }^{(12)}$. Além do mais, outros autores referem que além do estresse, a aplicação do OSCE está associada também a nervosismo e ansiedade ${ }^{(7,22)}$. Estes sentimentos podem ser ocasionados devido ter sido a primeira vez em que os estudantes foram expostos a esse tipo de método $\operatorname{avaliativo}^{(23)}$.
Porém, torna-se importante apontar que tais sentimentos referidos no momento avaliativo são diminuídos na prática nos estabelecimentos de saúde e este é um dos principais objetivos com a utilização do OSCE. Em consonância com tal assertiva, outro estudo(11) confirma que as simulações em laboratório reduzem o medo e a insegurança dos estudantes quando estes vão para a prática, além de facilitar a aprendizagem.

Vale salientar que apesar do estresse e ansiedade afetar negativamente o desempenho dos estudantes, estes sentimentos podem refletir o estresse vivido em um caso real, assim, esta dificuldade pode aumentar a validade da utilização do OSCE como estratégia de ensino ${ }^{(8)}$.

Outra categoria do segundo questionamento demonstrou por parte dos estudantes que o OSCE é uma experiência válida para a prática. Diante desta afirmação, um estudo ${ }^{(20)}$ revelou que é de suma importância para o desenvolvimento de habilidades o contato prévio do estudante com situações reais simuladas, com o intuito de adquirir segurança e diminuir o nervosismo durante a realização de procedimentos frente ao cliente. Assim, o estudante adquire habilidades antes de encarar o cuidado com o paciente no momento da prática em um estabelecimento de saúde.

Do mesmo modo, outros autores identificaram que a maioria dos estudantes referiu que o OSCE testa uma ampla área de conhecimentos e habilidades e que, além disso, ajuda a identificar as lacunas que necessitam ser melhoradas no aprendizado. Um grande número de participantes avaliou o OSCE como uma experiência prática valiosa para a aquisição de conhecimento, análogo ao estudo ora elaborado ${ }^{(5,24)}$.

A terceira categoria da segunda questão incluiu que por ser um método avaliativo, foi negativo no desempenho, acredita-se que a presença do professor no momento da avaliação gera nos estudantes os sentimentos de medo, nervosismo e ansiedade, os quais são decisivos no desempenho do estudante, conforme já 
discutido na primeira categoria do segundo questionamento.

Coerente a esta categoria, outros autores explanam em seu relato que a presença do professor no momento do OSCE apresenta desvantagens, que podem ser atenuadas, uma vez que podem dificultar o desempenho do estudante no momento da simulação do atendimento clínico. Porém, se o professor mantiver uma relação igualitária de críticas e elogios com o estudante, este pode facilitar e estimular o processo de ensinoaprendizagem $^{(3)}$.

Em contraponto, outro estudo ${ }^{(21)}$ refere que o OSCE tem sido considerada uma forma eficaz de avaliar a competência clínica de vários profissionais de saúde. Uma das principais vantagens do OSCE apontada é a possibilidade de ligar um conjunto de competências essenciais com diversas situações clínicas simuladas, de forma a permitir a mensuração de determinadas competências. Isto sugere que o OSCE pode ser considerado como um "padrão ouro" para avaliar futuros profissionais da saúde.

Segundo o Quadro 3, pôde-se observar que com a interpretação da terceira questão norteadora foram identificadas duas categorias. A primeira categoria deste questionamento demonstrou a vantagem da utilização deste método devido ao fato do estudante poder aprender com os erros. Relativo a isto, estudo ${ }^{(3)}$ expõe que com o uso desta estratégia, o estudante pode cometer erros sem gerar danos ao paciente real, uma vez que pode modificá-los. Ao identificar a falha, medidas são realizadas no intuito de evitar a repetição de tal deslize.

Outro autor comenta que o uso de simulações em laboratório é avaliado como um importante fator de melhora na atuação profissional, já que oferece a possibilidade de aprender com os erros. Da mesma forma, outra vantagem está em diminuir os erros iatrogênicos aos pacientes reais ${ }^{(20)}$.

A segunda categoria deste questionamento evidenciou que a semelhança com a prática é importante no momento do desempenho das atividades nos campos reais. Autores de um estudo sobre evolução das competências dos estudantes de medicina demonstraram que os problemas retratados no OSCE são aqueles em que os estudantes comumente encontram em estabelecimentos de saúde. Pacientes padronizados tipicamente têm queixas gerais, embora alguns possam apresentar problemas relacionados às condições de emergência ${ }^{(25)}$.

Concomitante, existem várias vantagens das simulações em laboratório, entre elas está a possibilidade do estudante treinar e repetir quantas vezes forem necessárias situações clínicas de maneira a adquirir segurança e habilidade durante as práticas hospitalares $^{(20)}$.

\section{CONCLUSÃO}

Assim, de acordo com as avaliações dos estudantes, - OSCE constituiu uma estratégia avaliativa e principalmente educativa de grande importância no processo dinâmico de ensino-aprendizagem.

Destaca-se que o uso desta estratégia apresenta pontos positivos e negativos. Porém, observa-se nas falas que as vantagens se sobressaem quando se compara ao pequeno número de pontos negativos. Dos pontos negativos destacam-se os principais sentimentos e o método avaliativo. Entre as questões positivas, evidenciase que a utilização do POP, a estratégia de aprendizagem, a experiência válida, aprender com os erros e a semelhança com a prática, foram apontadas pela maioria dos estudantes como de extrema importância antes da chegada destes aos campos de prática e para aquisição e desenvolvimento do conhecimento na saúde com ênfase na enfermagem.

Destarte, sugere-se que este método seja empregado em outras disciplinas e em outros cursos, principalmente na área da saúde, gerando assim a formação de profissionais cada vez mais habilitados e capacitados para atuarem na prática e realizarem um cuidado de qualidade ao cliente. 


\section{REFERÊNCIAS}

1. Illesca P. M, Cabezas G. M, Romo P. MT, Díaz R. P. Opinión de estudiantes de enfermería sobre el examen clínico objetivo estructurado. Cienc. enferm. [Internet]. 2012 [acesso em: 30 set 2015];XVIII(1):99-109. Disponível em: http://dx.doi.org/10.4067/S0717-95532012000100010. 2. Braccialli LAD, Oliveira MAC. Conceptions of performance evaluation in a competence-centered curriculum. Rev Esc Enferm USP [Internet]. 2011 [acesso em: 30 set 2015];45(5):1221-8. Disponível em: http://dx.doi.org/10.1590/S0080-62342011000500027. 3. Galato D, Alano GM, França TF, Vieira AC. Exame clínico objetivo estruturado (ECOE): uma experiência de ensino por meio de simulação do atendimento farmacêutico. Interface (Botucatu) [Internet]. 2011 [acesso em: 30 set 2015];15(36):309-20. Disponível em: http://dx.doi.org/10.1590/S1414-32832010005000032. 4. Turner JL, Dankoski ME. Objective Structured Clinical Exams: A Critical Review. Fam Med [Internet]. 2008 [acesso em: 30 set 2015];40(8):574-8. Disponível:

http://www.stfm.org/FamilyMedicine/Vol40Issue8/Turner574. 5. Selim AA, Ramadan FH, El-Gueneidy MM, Gaafer MM. Using Objective Structured Clinical Examination (OSCE) in undergraduate psychiatric nursing education: is it reliable and valid? Nurse Educ Today [Internet]. 2012 [acesso em: 30 set 2015];32(3):283-8. Disponível:

http://dx.doi.org/10.1016/j.nedt.2011.04.006.

6. Furlong $E$, Fox $P$, Lavin $M$, Collins R. Oncology nursing students' views of a modified OSCE. Eur J Oncol Nurs [Internet]. 2005 [acesso em: 30 set 2015];9(4):351-9. Disponível em: http://dx.doi.org/10.1016/j.ejon.2005.03.001.

7. Muldoon K, Biesty L, Smith V. 'I found the OSCE very stressful': student midwives' attitudes towards an objective structured clinical examination (OSCE). Nurse Educ Today [Internet]. 2014 [acesso em: 30 set 2015];34(3):468-73. Disponível em: http://dx.doi.org/10.1016/j.nedt.2013.04.022. 8. Paul F. An exploration of student nurses' thoughts and experiences of using a video-recording to assess their performance of cardiopulmonary resuscitation (CPR) during a mock objective structured clinical examination (OSCE). Nurse Educ Pract [Internet]. 2010 [acesso em: 30 set 2015];10(5):28590. Disponível em:

http://dx.doi.org/10.1016/j.nepr.2010.01.004.

9. Bowling AM. The effect of simulation on skill performance: a need for change in pediatric nursing education. J Pediatr Nurs [Internet]. 2015 [acesso em: 30 set 2015];30(3):439-46. Disponível em: http://dx.doi.org/10.1016/j.pedn.2014.12.010. 10. Santos IS, Vieira JE, Nunes MPT. Length of internship influences performance on Medical Residency exam. Rev Assoc Med Bras [Internet]. 2009 [acesso em: 30 set 2015];55(6):7448. Disponível em: http://dx.doi.org/10.1590/S010442302009000600021.

11. Mazzoni CJ, Moraes MAA. A avaliação prática estruturada de habilidades clínicas na Famema: fundamentos para construção e aplicação. Revista Gestão Universitária [Internet]. 2006 [acesso em: 30 set 2015]. Disponível em: http://gestaouniversitaria.com.br/artigos/a-avaliacao-pratica- estruturada-de-habilidades-clinicas-na-famema-fundamentospara-construcao-e-aplicacao.

12. Medeiros SB, Pereira CDFD, Tourinho FSV, Fernandes LGG, Santos VEP. Exame clínico objetivo estruturado: reflexões sob um olhar da enfermagem. Cogitare Enferm [Internet]. 2014 [acesso em: 30 set 2015];19(1):170-3. Disponível em: http://dx.doi.org/10.5380/ce.v19i1.35977.

13. Gamboa-Salcedo T, Martínez-Viniegra N, Peña-Alonso YR, Pacheco-Ríos A, García-Durán R, Sánchez-Medina J. Examen Clínico Objetivo Estructurado como instrumento para evaluar la competencia clínica en Pediatría. Estudio piloto. Bol. Med. Hosp. Infant. Mex. [Internet]. 2011 [acesso em: 30 set 2015];68(3):184-92. Disponível em:

http://www.scielo.org.mx/scielo.php?script=sci_arttext\&pid=S 1665-11462011000300003\&lng=es.

14. Santos AP, Vanderley TS, Brasileiro ME. Implementação da Sistematização de Assistência de Enfermagem em Parada Cardiorrespiratória na sala de emergência. Revista Eletrônica de Enfermagem do Centro de Estudos de Enfermagem e Nutrição [Internet]. 2012 [acesso em: 30 set 2015];3(3):116.Disponível em:

http://www.cpgls.pucgoias.edu.br/7mostra/Artigos/SAUDE\%2 0E\%20BIOLOGICAS/Implementação\%20da\%20SAE\%20em\%20P CR\%20na\%20sala\%20de\%20emergência.pdf.

15. Kuark FS, Manhães FC, Medeiros CH. Metodologia da pesquisa: guia prático. Itabuna: Via Litterarum; 2010.

16. Franco MLPB. Análise de Conteúdo. 2st ed. Brasília: Liber Livro Editora; 2005.

17. Resolução № 466 do Conselho Nacional de Saúde, de 12 de dezembro de 2012 (BR) [Internet]. Aprova as diretrizes e normas regulamentadoras de pesquisas envolvendo seres humanos. Diário Oficial da União. 13 jun. 2013 [acesso em: 30 set 2015]. Disponível em:

http://bvsms.saude.gov.br/bvs/saudelegis/cns/2013/res0466 12_12_2012.html.

18. Honório RPP, Caetano JA, Almeida PC. Validação de procedimentos operacionais padrão no cuidado de enfermagem de pacientes com cateter totalmente implantado. Rev Bras Enferm [Internet]. 2011 [acesso em: 30 set 2015];64(5):882-9. Disponível em: http://dx.doi.org/10.1590/S0034-71672011000500013. 19. Barbosa CM, Mauro MFZ, Cristóvão SAB, Mangione JA. A importância dos procedimentos operacionais padrão (POPs) para os centros de pesquisa clínica. Rev Assoc Med Bras [Internet]. 2011 [acesso em: 30 set 2015];57(2):134-5. Disponível em: http://dx.doi.org/10.1590/S010442302011000200007.

20. Silveira RCP, Robazzi MLCC. Modelos e inovações em laboratórios de ensino em enfermagem. Revista de Enfermagem do Centro-Oeste Mineiro [Internet]. 2011 [acesso em: 30 set 2015];1(4):592-602. Disponível em:

http://www.seer.ufsj.edu.br/index.php/recom/article/viewArti cle/138.

21. Silva CCBM, Lunardi AC, Mendes FAR, Souza FFP, Carvalho CRF. Objective structured clinical evaluation as an assessment method for undergraduate chest physical therapy students: a cross-sectional study. Rev. bras. fisioter. [Internet]. 2011 
[acesso em: 30 set 2015];15(6):481-6. Disponível em:

http://dx.doi.org/10.1590/S1413-35552011005000033.

22. Nulty DD, Mitchell ML, Jeffrey CA, Henderson A, Groves M.

Best Practice Guidelines for use of OSCEs: Maximising value for student learning. Nurse Educ Today [Internet]. 2011 [acesso em: 30 set 2015];31(2):145-51. Disponível em:

http://dx.doi.org/10.1016/j.nedt.2010.05.006.

23. Nasir AA, Yusuf AS, Abdur-Rahman LO, Babalola OM, Adeyeye AA, Popoola AA et al. Medical students' perception of objective structured clinical examination: a feedback for process improvement. J Surg Educ [Internet]. 2014 [acesso em: 30 set 2015];71(5):701-6. Disponível em:

http://dx.doi.org/10.1016/j.jsurg.2014.02.010.

24. Branch C. An assessment of students' performance and satisfaction with an OSCE early in an undergraduate pharmacy curriculum. Curr Pharm Teach Learn [Internet]. 2014 [acesso em: 30 set 2015];6(1):22-31. Disponível em: http://dx.doi.org/10.1016/j.cptl.2013.09.006. 25. Hsieh M-C, Cheng W-C, Chen T-Y. Objective Structured Clinical Examination (OSCE) including critical simulation: Evaluation of medical student competence. Tzu Chi Med J [Internet]. 2014 [acesso em: 30 set 2015];26(1):40-3. Disponível em: http://dx.doi.org/10.1016/j.tcmj.2013.08.001.

Recebido: 13/12/2014.

Aceito: $12 / 05 / 2015$.

Publicado: 30/09/2015. 